\title{
Silencing of long non-coding RNA PCAT6 restrains gastric cancer cell proliferation and epithelial-mesenchymal transition by targeting microRNA-15a
}

\author{
Dongfang Dong, Yue Lun, Bo Sun, Haiyuan Sun, Qunying Wang, Gang Yuan and Jingzi Quan \\ Department of Gastroenterology, No.971 Hospital of People's Liberation Army Navy, Qingdao, China
}

\begin{abstract}
Gastric cancer (GC) is a high mortality disease. We studied the function and mechanism of long non-coding RNA prostate cancer-associated transcript 6 (lncRNA PCAT6) on cell proliferation and epithelial-mesenchymal transition (EMT) in GC cells. CCK-8, flow cytometry and colony formation assay were respectively used to detect the cell viability, apoptosis and colony formation. PCAT6 and miR-15a expression were changed by cell transfection. Moreover, the level of Cyclin D1, p53, Bax, Cleaved caspase-3 and relate-proteins of EMT and cell pathways were investigated by Western blot. Besides, the level of miR-15a and PCAT6 was tested by RT-qPCR. Besides, the target relation between miR-15a and PCAT6 were tested by luciferase assay. PCAT6 was highly expressed in GC cells and tissues. Silencing of PCAT6 restrained the relate-proteins of cell proliferation and EMT. Furthermore, PCAT6 reversely regulated miR-15a and miR-15a inhibitor reversed the efficacy of sh-PCAT6 in cell proliferation and EMT. PCAT6 restrained the relate-proteins of RB/E2F and Wnt $/ \beta$-catenin pathways and miR-15a reverse this progress. Finally, PCAT6 was a target of miR-15a. Silencing of IncRNA PCAT6 restrained proliferation and EMT of GC cells by targeting miR-15a via $\mathrm{RB} / \mathrm{E} 2 \mathrm{~F}$ and $\mathrm{Wnt} / \beta$-catenin pathways.
\end{abstract}

Key words: Gastric cancer - EMT — lncRNA PCAT6 - miR-15a

\section{Introduction}

Gastric cancer (GC) is a malignant tumor derived from the gastric mucosal epithelium. It has a high incidence all over the world (Karimi et al. 2014). Due to the changes in dietary structure, increased work pressure and other reasons, GC has squinted towards younger. The current treatment methods mainly cover surgery, chemotherapy and drug treatment, but the forms and functions of GC drugs are finitude now (Lordick et al. 2017; Tsukamoto et al. 2017). Most of the inchoate stages of GC have no obvious symptoms, and the inchoate diagnosis rate is still low (Song et al. 2017b). Therefore, strengthening the research on the pathogenesis of GC is of great significance for the prevention of GC.

Long non-coding RNA (lncRNA) is a class of RNAs that have no protein-coding potential, over $200 \mathrm{nt}$ in length (Jar-

Correspondence to: Jingzi Quan, Department of Gastroenterology, No. 971 Hospital of People's Liberation Army Navy, No. 22, Minjiang Road, Shinan District, Qingdao 266071, China E-mail: jingzq0114@sina.com roux et al. 2017). Studies had displayed that lncRNAs played a momentous part in the progress of GC (NasrollahzadehKhakiani et al. 2017). PCAT6 (prostate cancer associated transcript 6) is a newly discovered carcinogenic lncRNA. Studies proved PCAT6 enhanced cell proliferation in prostate tumor (Musumeci et al. 2011) and enhanced cell proliferation and metastasis in lung cancer by regulating miR-330-5p (Cui et al. 2018). At the same time, a research had confirmed that PCAT6 was highly expressed in GC and regulated miR-30 in tumor (Xu et al. 2018).

MicroRNA (miRNA) is a non-coding RNA of approximately 21-25 nt in size (Hrovatin et al. 2018). Many miRNAs have been found to play momentous parts in GC (Shin et al. 2014). Studies had proved miR-15a was lower express in GC (Wu et al. 2016). Besides, miR-15a had been displayed to play an inhibitory action in GC (Kang et al. 2015). Therefore, miR-15a may be a potential treatment regimen in GC.

In this study, we focused on the function of PCAT6 in GC. Firstly, we investigated the standard of PCAT6 in GC tissues and GC cells. Besides, we selected the two cells with the highest expression. Transforming growth factor $\beta 1$ (TGF- $\beta 1$ ) 
was used to cause the epithelial-to-mesenchymal transition (EMT) in vitro. The level of PCAT6 was further examined to determine whether there was a correlation between PCAT6 and miR-15a. Besides, we investigated the possible internal molecular mechanisms mediated by PCAT6. This study may help to understand the role of PCAT6 and provide new target for the diagnosis and treatment of GC.

\section{Materials and Methods}

\section{Ethics approval}

All procedures performed in studies involving human participants were in accordance with the ethical standards of the institutional committee and with the 1964 Helsinki declaration and its later amendments or comparable ethical standards.

\section{Clinical specimens}

Clinical human GC tissues and the correlative adjacent tissues $(n=20)$ were acquired from No. 971 Hospital of People's Liberation Army Navy (Qingdao, China). The patients did not acquire any treatments before surgery. Every patient agreed with joining the research and writing informed consent, and this research was ratified by the Medical Ethics Committee of No. 971 Hospital of People's Liberation Army Navy.

\section{Cell culture and treatment}

Human GC cells MKN45, SGC-7901, AGS, MKN28 and gastric epithelial cell GES-1 (China Center for Type Culture Collection, CCTCC, Wuhan, China) were cultured in Dulbecco's modified Eagle's medium (DMEM, Gibco, Grand Island, NY, US). The medium containing $10 \%$ fetal bovine serum (FBS, Gibco, US) $100 \mathrm{U} / \mathrm{ml}$ penicillin (Beyotime, Haimen, China) and $100 \mu \mathrm{g} / \mathrm{ml}$ streptomycin (Beyotime, China) at $5 \% \mathrm{CO}_{2}$, $37^{\circ} \mathrm{C}$. The culture medium was replaced $2-4$ days to achieve confluence. TGF- $\beta 1$ (Abcam, Cambridge, UK) was reconstituted with $\mathrm{ddH}_{2} \mathrm{O}, 0.1 \%$ albumin from bovine serum (BSA, Beyotime, US) to $10 \mathrm{ng} / \mathrm{ml}$. Cells were host starvation $8 \mathrm{~h}$, after added TGF- $\beta 1$ (10 ng/ml) $12 \mathrm{~h}$ to cause EMT.

\section{CCK-8 assay}

Cells were inoculated in 96-well plates (FPT011, Beyotime, China) at a $5 \times 10^{3}$ cells/well. Cells $\left(2 \times 10^{3}\right.$ cells/well $)$ were cultured at $5 \% \mathrm{CO}_{2}$ at $37^{\circ} \mathrm{C}$. After transfecting negative control (NC) miRNA and lncRNA, PCAT6 and miR-15a inhibitor, the 10 ul CCK- 8 reagent (C0038, Beyotime, China) was added to the medium. The plates were incubated $1 \mathrm{~h}$ in the dark at $5 \% \mathrm{CO}_{2}, 37^{\circ} \mathrm{C}$. The absorbance was quantitated by microplate reader (Bio-Rad, Sunnyvale, CA, US) at $450 \mathrm{~nm}$.

\section{Colony formation assay}

500 viable cells were inoculated in 6-well plates (Beyotime, China) for $24 \mathrm{~h}$. The cells were washed with phosphate buffered saline (PBS) and cultured in complete medium at $5 \% \mathrm{CO}_{2}$ at $37^{\circ} \mathrm{C}$ for 10 days. The resulting colonies were fixed with 10\% formalin (G2161, Solarbio, Beijing, China) and stained with $0.1 \%$ crystal violet (C0121, Beyotime, China) for $10 \mathrm{~min}$. Each treatment run triplicate. Colonies were counted and compared to untreated cells (a clone was regarded as $>50$ cells).

\section{Flow cytometry}

Flow cytometry was carried out with PI and FITC-conjugated annexin V staining (C1062M, Beyotime, China). PBS washed the fixed cells twice and PI/FITC-Annexin $\mathrm{V}$ stained, followed by hatch in the dark at $1 \mathrm{~h}$. This assay was implemented by FACS can (Beckman Coulter, Fullerton, CA, USA). The data was analyzed by FlowJo software (Tree Star Software, San Carlos, California, US).

\section{Reverse transcription quantitative PCR (RT-qPCR)}

Trizol was used to extract RNA (Molecular Research Center, Cincinnati, Ohio). The Taqman MicroRNA Reverse Transcription Kit (4366597, Thermo Fisher Scientific, Runcorn, UK) and Taqman Universal Master Mix II within TaqMan MicroRNA Assay of miR-15a and U6 (Thermo Fisher Scientific, US) were used for detecting miR-15a expression. SYBR ${ }^{\circledR}$ PrimeScript ${ }^{\oplus}$ PLUS RT-RNA PCR Kit (RR037B, TaKaRa, China) was used for the Real-Time PCR analysis to test the level of PCAT6, GAPDH was an internal parameters. Samples were run in triplicate. The $2_{-\Delta \Delta \mathrm{Ct}}$ equation was used to quantify the data.

\section{Transfection}

miR-15a inhibitor is a chemically modified single strand RNA that can competitively bind to mature miR-15a sequences. It can specifically silence miR-15a. Short hairpin (sh) RNA targeting lncRNA PCAT6 were bind to U6/GFP/ Neo plasmid (GenePharma, Shanghai, China), and called sh-PCAT6. The miR-15a inhibitor and the negative control (NC) miRNA inhibitor were synthesized by GenePharma Co. (Shanghai, China). Lipofectamine 3000 (Carlsbad Life Technologies, Carlsbad, CA, US) was used on cell transfection. Stably transfected cells were selected by using medium which was containing $0.5 \mathrm{mg} / \mathrm{ml} \mathrm{G} 418$ (Sigma, US). After about 4 weeks, G418 resistant cells were successfully estab- 
lished. Since the highest transfection efficiency occurred at the $48 \mathrm{~h}$, the harvest time was $72 \mathrm{~h}$ after transfection in the subsequent experiments.

\section{Western blot}

Proteins were extracted from cells by RIPA lysis buffer (R0010, Solarbio, China) plus protease inhibitor (A8260, Solarbio, China). We used protein assay kit (PC0020, Solarbio, China) quantified the proteins. This experiment was established by using the Bio-Rad system. Primary antibodies (Abcam,US) contained Cyclin D1 (ab16663), p53 (ab131442), Bax (ab32503), pro Caspase-3 (ab32499), Cleaved Caspase-3 (ab32042), $\beta$-actin (ab8227), RB (ab181616), p-RB (ab47763), E2F (ab179445), Wnt3a (ab28472), $\beta$-catenin (ab16051), E-cadherin (E-cad) (ab15148), N-cadherin (N-cad) (ab18203), Vimentin (ab137321), Snail (ab82846) and zinc-finger E-box binding homeobox 1 (ZEB1) (ab124512). Primary antibodies were prepared at a dilution of 1:1000 in 5\% blocking buffer (BSA, SW3015, Solarbio, China). The primary antibodies were cultured at $4^{\circ} \mathrm{C}$ overnight, washed and incubated with secondary antibodies goat anti-rabbit IgG (ab6721, 1:5000) $1 \mathrm{~h}$ at $25^{\circ} \mathrm{C}$. After rinsing, the polyvinylidene fluoride (PVDF) membrane (FFP36, Beyotime, China) bring the antibody were shifted to system. Captured signal and quantified by Image Lab ${ }^{\text {Tw }}$ Software (Bio-Rad, US). The above experiments were used $\beta$-actin as internal parameters.

\section{Statistical analysis}

All experiments were repeated three times. Data were expressed as mean \pm standard deviation (SD). Statistical analyses were performed by using Graphpad 6.0 (Graph Pad Software, CA, US). The $p$-values were calculated using a one-way analysis of variance (ANOVA) and $t$-test. $p<0.05$ was considered statistically significant.

\section{Results}

PCAT6 was highly expressed in GC cells and tissues

RT-qPCR was used to investigate the level of PCAT6 in GC patients, corresponding normal gastric tissues and cells. The results revealed that the level of PCAT6 was prominently higher in GC tissues than that in normal tissues (Fig. 1A, $p<0.001)$. The results also revealed that PCAT6 was highly expressed in MKN45, SGC-7901, AGS, and MKN28 cells (Fig. $1 \mathrm{~B}, p<0.05$ or $p<0.001$ ). In the next experiment, we selected MKN45 and SGC-7901 cells with the highest expression to investigate the functions of PCAT6.

\section{Silencing of PCAT6 restrained cell proliferation}

The Fig. 2A $(p<0.01)$ displayed that sh-PCAT6 can conspicuously decline the expression of PCAT6 in MKN45 and SGC-7901 cells. This implied transfection efficiency was high. We explored the functions of PCAT6 from the level of cellular and molecular in MKN45 and SGC-7901 cells. sh-PCAT6 meaningfully restrained cell viability (Fig. 2B, $p<0.05$ or $p<0.01$ ) and colony ability (Fig. 2 C, $p<0.01$ ), meanwhile, it meaningfully enhanced cell apoptosis (Fig. $2 \mathrm{D}, p<0.001)$. At the molecular level, when sh-PCAT6 was transfected, Western blot results (Fig. 2E-G) displayed that the level of the Cyclin D1 was conspicuously declined and
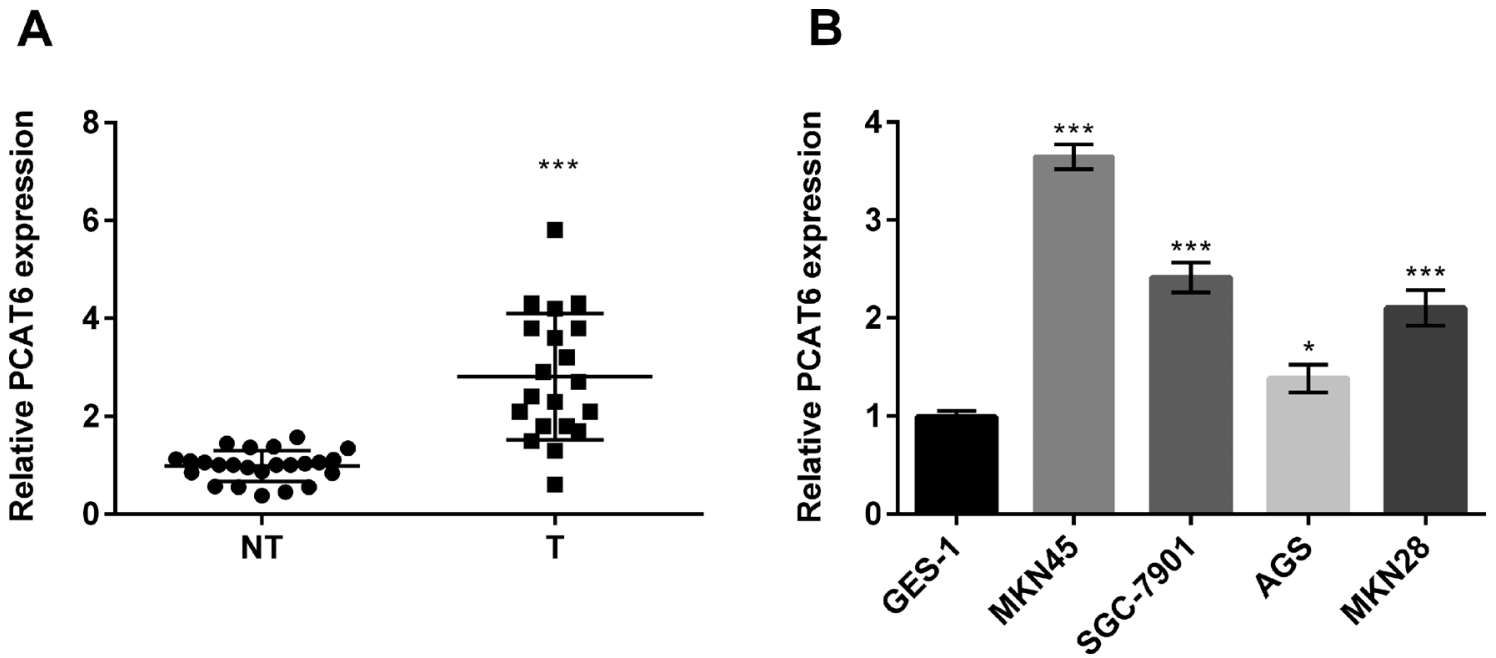

Figure 1. Prostate cancer-associated transcript 6 (PCAT6) was highly expressed in gastric cancer tissue (A) and in MKN45, SGC-79017901, AGS and MKN28 cells (B). ${ }^{*} p<0.05 ;{ }^{* *} p<0.001$. NT, non tumor; T, tumor. 


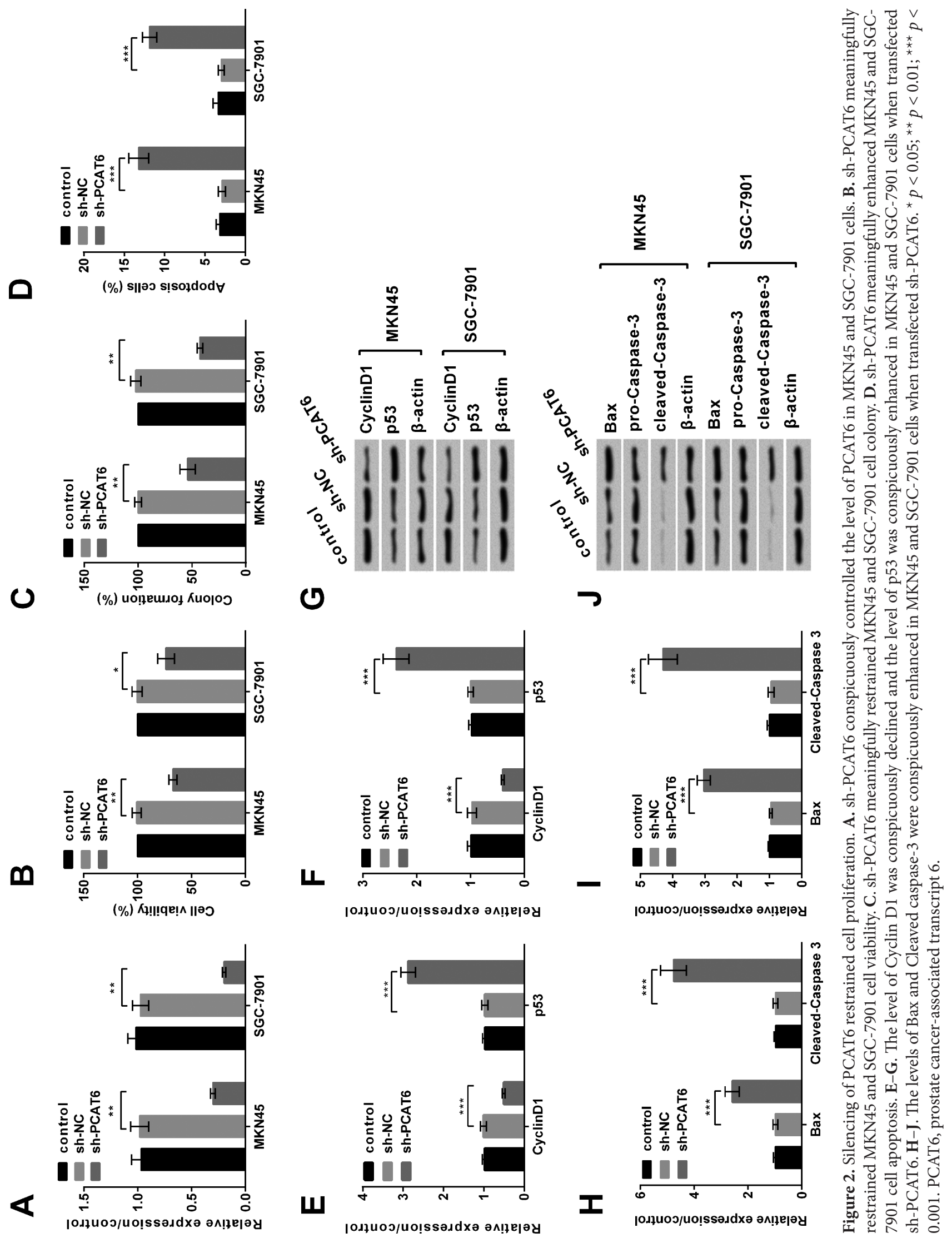




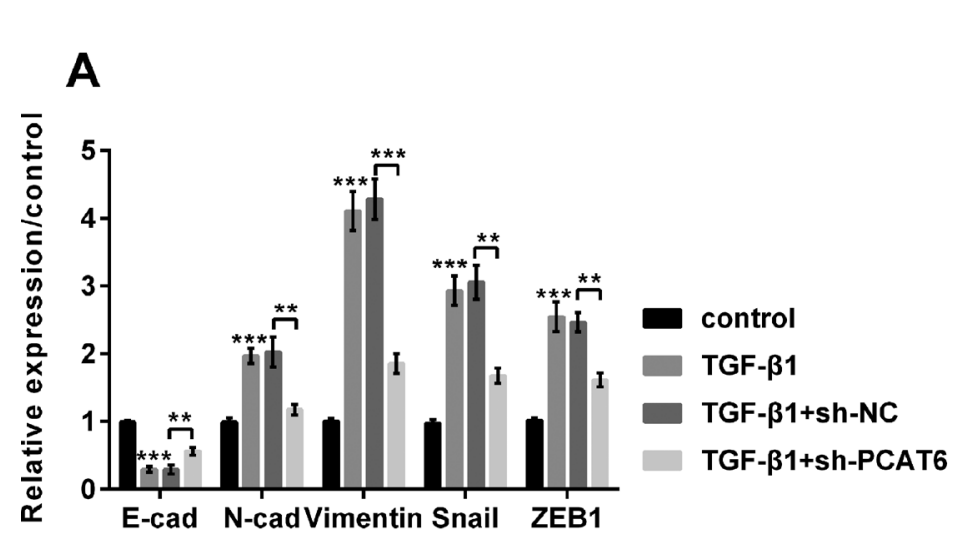

B
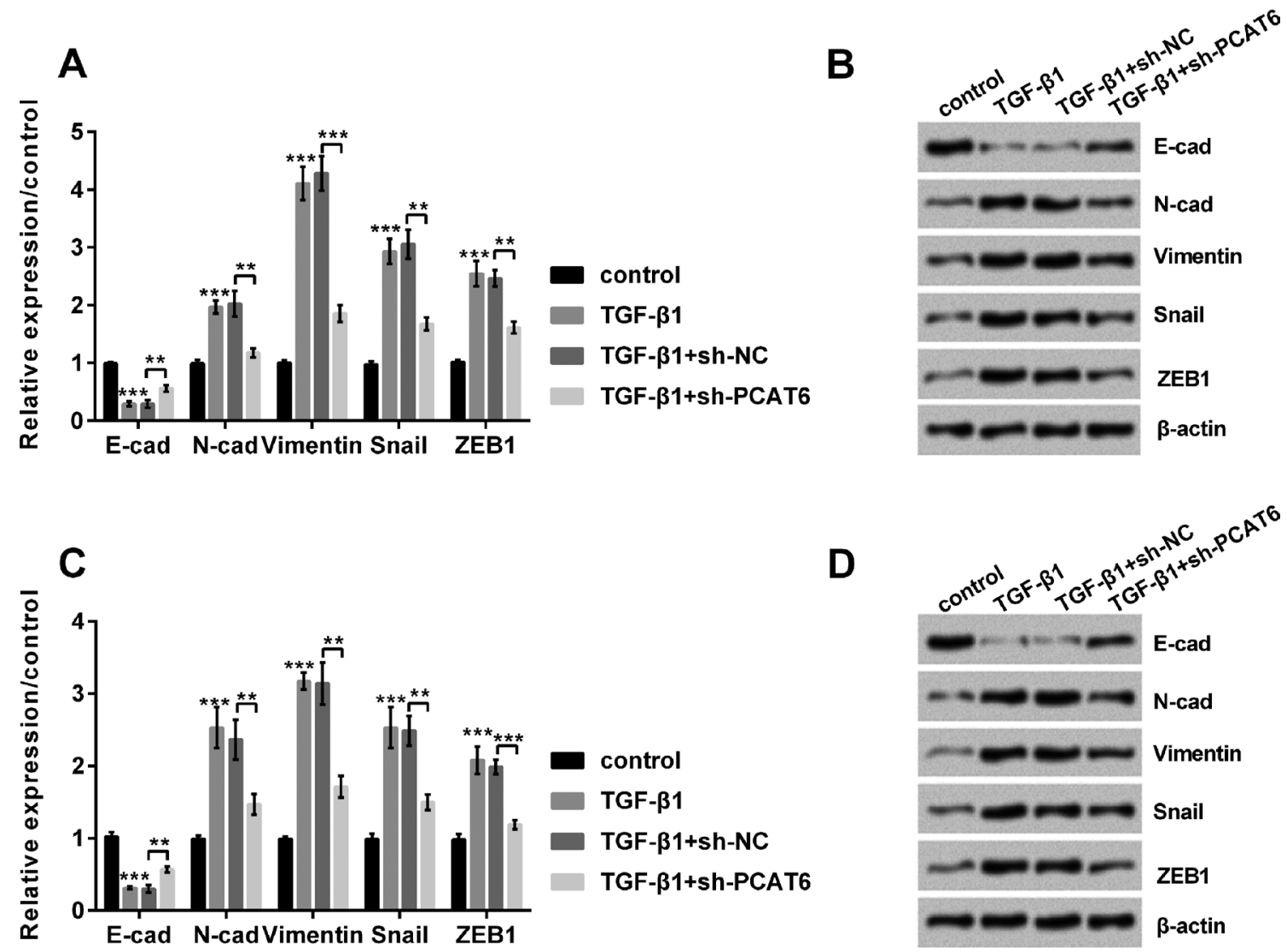

Figure 3. Silencing of PCAT6 restrained the cell epithelial-mesenchymal transition. TGF- $\beta 1$ conspicuously declined the level of E-cad and enhanced the levels of N-cad, Vimentin, Snail and ZEB1, and sh-PCAT6 conspicuously enhanced the level of E-cad and declined the levels of N-cad, Vimentin, Snail and ZEB1 which was caused by TGF- $\beta 1$ in MKN45 cells (A and B) and in SGC-7901 cells (C and D). ${ }^{* *} p<0.01 ;{ }^{* * *} p<0.001$. PCAT6, prostate cancer-associated transcript 6; TGF- $\beta 1$, transforming growth factor $\beta 1$; E-cad, E-cadherin; $\mathrm{N}$-cad, N-cadherin; ZEB1, zinc-finger E-box binding homeobox 1.

p53 was conspicuously enhanced. Fig. $2 \mathrm{H}-\mathrm{J}$ results proved the levels of Bax and Cleaved caspase- 3 were also conspicuously increased. In conclusion, silencing of PCAT6 restrains cell proliferation in GC cells.

\section{Silencing of PCAT6 restrained the cell EMT}

Western blot was used for investigating the functions of PCAT6 on EMT in MKN45 and SGC-7901 cells. From Fig. $3 \mathrm{~A}$ and $\mathrm{B}$, TGF- $\beta 1$ conspicuously declined the level of E-cad $(p<0.001)$ and enhanced the levels of $\mathrm{N}$-cad, Vimentin, Snail and ZEB1 $(p<0.001)$ in MKN45 cells. On the other hand, sh-PCAT6 conspicuously enhanced the level of E-cad $(p<$ $0.01)$ and declined the levels of $\mathrm{N}-\mathrm{cad}(p<0.01)$, Vimentin $(p<0.001)$, Snail $(p<0.01)$ and ZEB1 $(p<0.01)$ which was caused by TGF- $\beta 1$ in MKN45 cells. The same results were displayed in SGC-7901 cells (Fig. 3C and D). Above results implied that silencing of PCAT6 restrains the EMT in GC cells.

\section{Silencing of PCAT6 up-regulated miR-15a}

The level of miR-15a was meaningfully enhanced after transfecting sh-PCAT6 in MKN45 and SGC-7901 cells (Fig. 4, $p<0.001)$. This implied PCAT6 was negatively regulated miR-15a.

Silencing of PCAT6 restrained cell proliferation by targeting miR-15a

Firstly, we transferred with NC inhibitor and miR-15a inhibitor into MKN45 and SGC-7901 cells. We found miR-15a inhibitor statistically declined miR-15a expression and this implied that we successfully transfected miR-15a inhibitor into the cell (Fig. 5A, $p<0.001$ ). Further results revealed that miR-15a inhibitor reversed the sh-PCAT6-induced decline in cell viability and cell colony ability, meanwhile, it declined and rise in cell apoptosis (Fig. 5B-D, $p<0.05$ ) in MKN45 and SGC-7901 cells. On the other hand, at the 


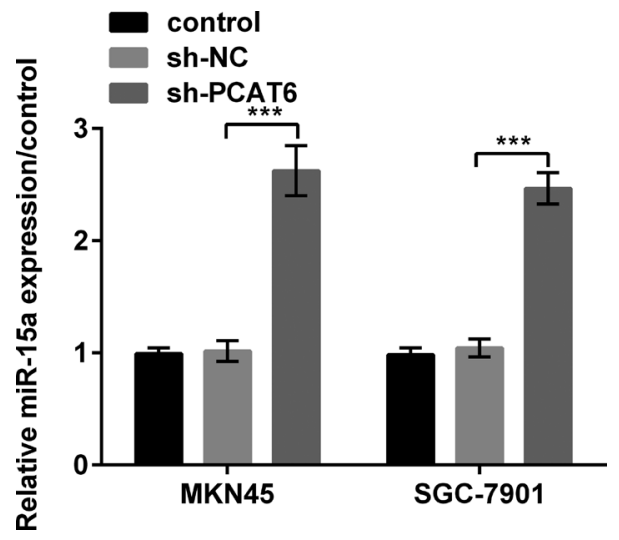

Figure 4. Silencing of PCAT6 up-regulated miR-15a. The level of miR-15a was meaningfully declined after the transfection of sh-PCAT6 in MKN45 and SGC-7901 cells. ${ }^{* *} p<0.001$. PCAT6, prostate cancer-associated transcript 6; miR, microRNA.

molecular level, Western blot results (Fig. 5E-G) displayed that the level of Cyclin D1 $(p<0.001)$ was conspicuously increased and p53 $(p<0.05$ or $p<0.01)$ was conspicuously declined when transfected miR-15a inhibitor, compared to transfection of sh-PCAT6 alone. Fig. 5H-J results proved the levels of Cleaved caspase- $3(p<0.05$ or $p<0.01)$ and Bax $(p<0.01$ or $p<0.001)$ which was caused by sh-PCAT6 were conspicuously declined when transfected miR-15a inhibitor. In short, silencing of PCAT6 restrained cell proliferation and promotes apoptosis by targeting miR-15a in GC.

\section{Silencing of PCAT6 restrained EMT by targeting miR-15a}

Western blot was used to investigate the efficacy of miR-15a on EMT in MKN45 and SGC-7901 cells. From the Fig. 6A and B, sh-PCAT6 conspicuously enhanced the level of E-cad $(p<0.001)$ and declined the levels of N-cad $(p<0.001)$, Vimentin $(p<0.001)$, Snail $(p<0.01)$ and ZEB1 $(p<0.001)$ in MKN45 cells. Meanwhile, miR-15a conspicuously declined sh-PCAT6-induced the level of E-cad $(p<0.001)$ and enhanced the levels of N-cad $(p<0.01)$, Vimentin $(p<0.01)$, Snail $(p<0.01)$ and ZEB1 $(p<0.01)$ in MKN45 cells. The same results were discovered in SGC-7901 cells (Fig. 6C and D). Above results implied that silencing of PCAT6 restrains EMT by targeting miR-15a.

\section{Silencing of PCAT6 restrained the RB/E2F and Wnt/ $\beta$-catenin signaling pathways via targeting miR-15a}

From the figure we can see that sh-PCAT6 enhanced the ratio of $\mathrm{pRB} / \mathrm{RB}$ ) and restrained the level of E2F (Fig. 7A and B, $p<0.001$ ), Wnt3a and $\beta$-catenin (Fig. 7E and F, $p<0.001$ ) in MKN45 cells. These expressions were reversed after the system was transfected with miR-15a inhibitor. The same tendency of pRB/RB and E2F (Fig. 7C and D, $p<0.001$ ), Wnt3a and $\beta$-catenin (Fig. $7 \mathrm{G}$ and $\mathrm{H}, p<0.01$ ) were found in SGC-7901 cells. Above results implied that silencing of PCAT6 restrained the $\mathrm{RB} / \mathrm{E} 2 \mathrm{~F}$ and $\mathrm{Wnt} / \beta$-catenin signaling pathways via targeting miR-15a.

\section{PCAT6 was a target of $m i R-15 a$}

To further investigate the relationship between miR-15a and PCAT6, we transferred miR-15a mimic into the luciferase system containing the target gene PCAT6. It displayed PCAT6 level was declined in PCAT6-WT and basically no change in PCAT6-MUT, indicating miR-15a is a target of PCAT6 ( $p<0.05$, Fig. 8A). The targeting sequence was shown in Fig. 8B.

\section{Discussion}

GC is a familiar malignant tumor across the globe with poor prognosis and high mortality (Hamashima 2014). In this study, firstly, we presented that PCAT6 was highly expressed in GC cells and tissues. Besides, we discovered that silencing of PCAT6 meaningfully restrained EMT and cell proliferation. Besides, we proved sh-PCAT6 negatively regulated miR-15a expression, silencing of PCAT6 restrained EMT and cell proliferation by targeting miR-15a. Finally, we discovered that PCAT6 exerted its efficacy by restraining the $\mathrm{RB} / \mathrm{E} 2 \mathrm{~F}$ and $\mathrm{Wnt} / \beta$-catenin signaling pathways.

EMT is foundational physiological phenomena. It can invade tumors (Pillai et al. 2015). When EMT occurs in the cells, the epithelial marker molecules E-cad is downregulated and the mesenchymal markers like Vimentin and $\mathrm{N}$-cad are up-regulated (Singh et al. 2018). The above marker molecules are regulated by transcription factors including the Snail and ZEB families. They restrain the level of E-cad by transcription, up-regulate the levels of mesenchymal markers, and initiate the EMT (Kim et al. 2012). TGF- $\beta 1$ universally exists in a variety of cells. It can play a significant part on impacting cell proliferation, differentiation and so on (Kajdaniuk et al. 2013). TGF- $\beta 1$ can straightforwardly act on cancer cells and cause EMT in cells, enhancing the augment of malignancy (Fabregat et al. 2014). A large number of studies had put into use TGF- $\beta 1$ to induce EMT (Bi et al. 2012; Mutlu et al. 2016; O'Leary et al. 2018). In this experiment, we displayed that TGF- $\beta 1$ conspicuously declined the level of E-cad and enhanced the levels of N-cad, Vimentin, Snail and ZEB1 in MKN45 and SGC-7901 cells. This means that we had successfully established the EMT model.

In recent years, studies had put forward that PCAT6 played a significant efficacy in the progression of cancer (Shi et al. 2018). Researchers displayed that PCAT6 was highly expressed in lung tumor and knockdown of PCAT6 


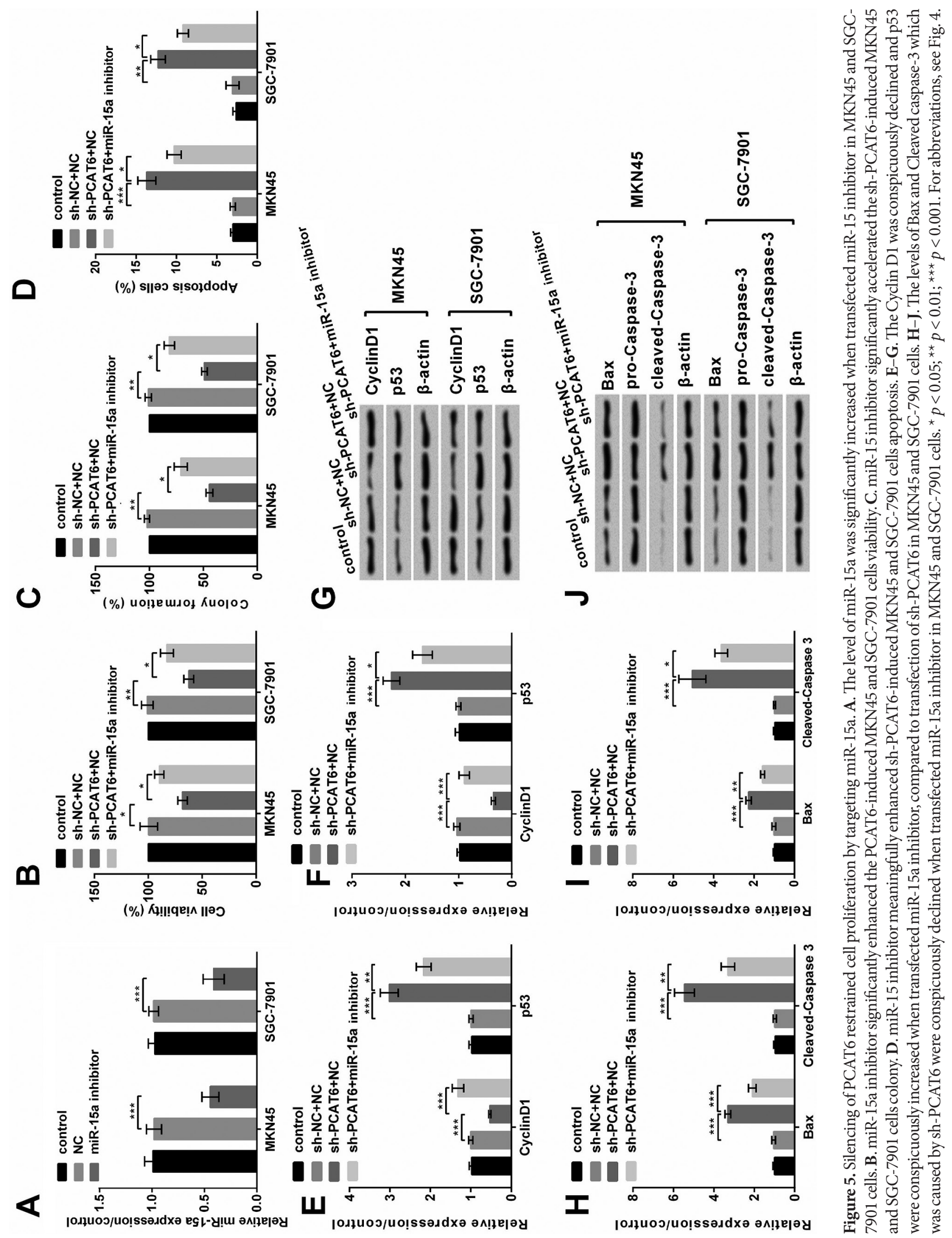




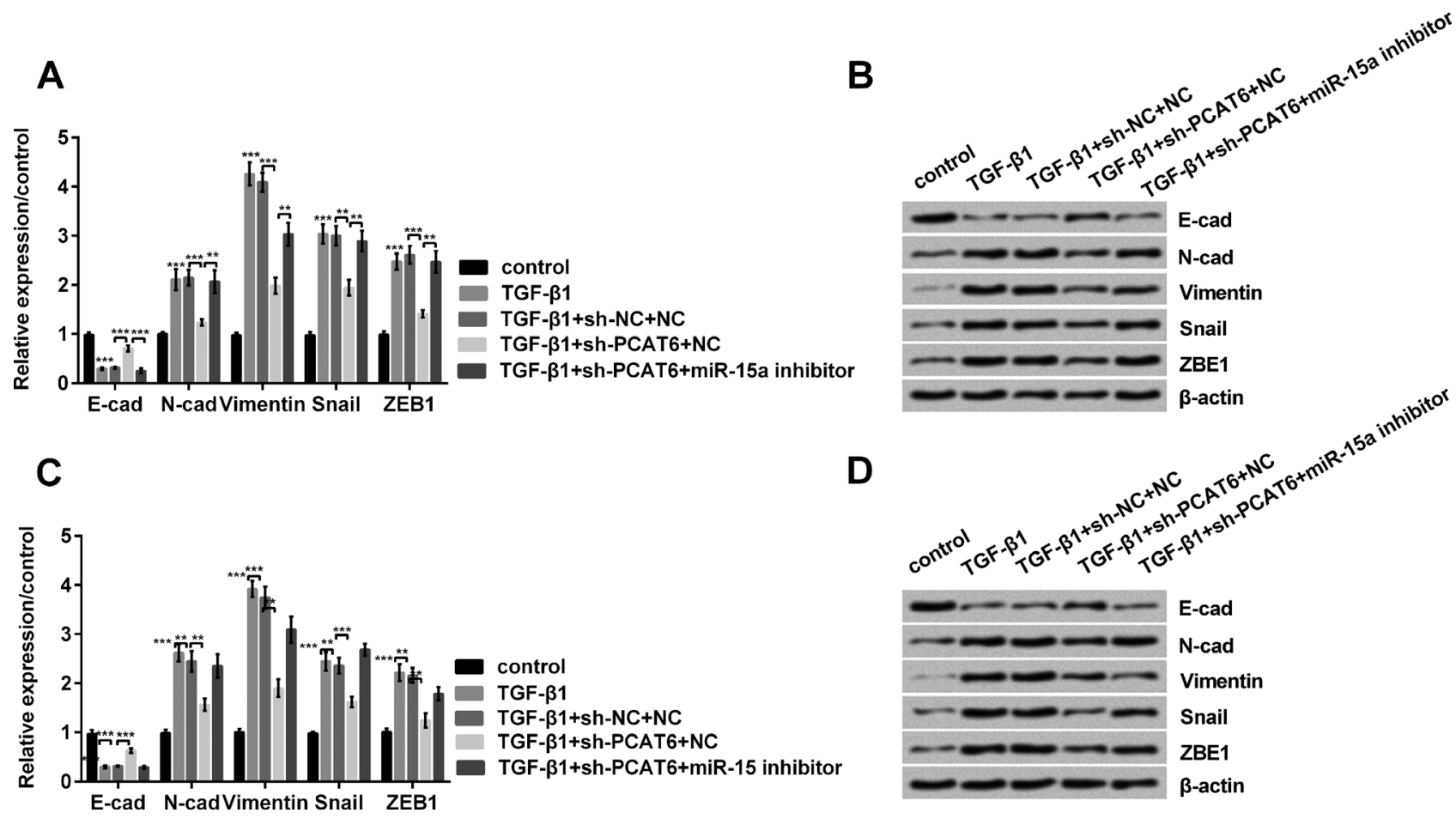

Figure 6. Silencing of PCAT6 restrained epithelial-mesenchymal transition by targeting miR-15a. sh-PCAT6 conspicuously enhanced the level of E-cad and declined the levels of N-cad, Vimentin, Snail and ZEB1; and miR-15a conspicuously declined sh-PCAT6-induced the level of E-cad and enhanced the levels of N-cad, Vimentin, Snail and ZEB1 in MKN45 cells (A and B) and in SGC-7901 cells (C and D). ${ }^{* *} p<0.01 ;{ }^{* *} p<0.001$. For abbreviations, see Fig. 3 .

inhibited proliferation and invasion in lung cancer cells (Wan et al. 2016). In addition, PCAT6 might be a tumor marker for lung cancer (Wan et al. 2017). Besides, studies proved PCAT6 worked in prostate tumor (Misawa et al. 2017) and could control liver tumor (Chen et al. 2019). Another study discovered that PCAT6 inhibited colon tumor by enhancer of zeste homolog 2 (EZH2) (Huang et al. 2019). Recently, a study confirmed that PCAT6 was highly expressed in GC (Xu et al. 2018). Our research was in line with the above researches, implying the tumor promoter function of PCAT6. Our results also displayed that PCAT6 was elevated in GC tissues and silencing of PCAT6 could inhibit cell proliferation and decline EMT.

Increasing studies suggested that lncRNAs exerted their functions by sponging miRNAs (Ling 2016). Previous studies verified that lncRNAs played a part on GC through miRNAs (Xia et al. 2014). For instance, lncRNA H19 worked in GC via miR-141 (Zhou et al. 2015) and lncRNA XIST enhanced GC via miR-497 (Ma et al. 2017). Further research displayed that PCAT6 exerted its function via miR-204 (Wu et al. 2019). Therefore, we hypothesized that PCAT6 might also be achieved by regulating miRNAs in GC. miR-15a can induce apoptosis, and also participates in tumor cell cycle through other genes (Cimmino et al. 2005). A research displayed that
miR-15a exerted functions in prostate tumor (Takeshita et al. 2010). Besides, there was also a study put forward that miR-15a modulated BCL-2 to exert functions (Cittelly et al. 2010). In addition, a literature discovered that miR-15a exerted efficacy in restraining GC (Song et al. 2017a). On the other hand, miR-15a can restrain the metastasis of GC cells by modulating Twist1 (Wang et al. 2017). Therefore, we further studied the relationship between PCAT6 and miR$15 \mathrm{a}$ in GC. Interestingly and similarly, in the current study, we demonstrated that miR-15a and PCAT6 were negatively correlated, and PCAT6 can participate in cell proliferation and EMT by regulating miR-15a in MKN45 and SGC-7901 cells. The functions of PCAT6 were reversed by miR-15 inhibitor in GC. Moreover, we further discovered PCAT6 was a target of miR-15a

The $\mathrm{RB} / \mathrm{E} 2 \mathrm{~F}$ and $\mathrm{Wnt} / \beta$-catenin signaling pathways play momentous parts in tumor (Schwarzenbach 2016). Previous studies had reported that $\mathrm{RB} / \mathrm{E} 2 \mathrm{~F}$ and $\mathrm{Wnt} / \beta$-catenin pathways were activated in GC and the two pathways were involved in the pathogenesis of GC (Birkenkamp-Demtroder et al. 2011). Studies proved TGF- $\beta 1$ caused EMT via RB/E2F and $W n t / \beta$-catenin signaling pathways (Dong et al. 2018) and PCAT6 exerted its efficacy via Wnt/ $\beta$-catenin signaling pathways (Singh et al. 2018). On the other hand, miR-15a was 
A

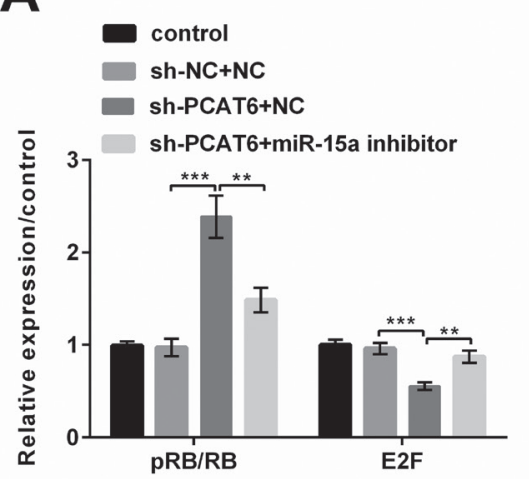

C

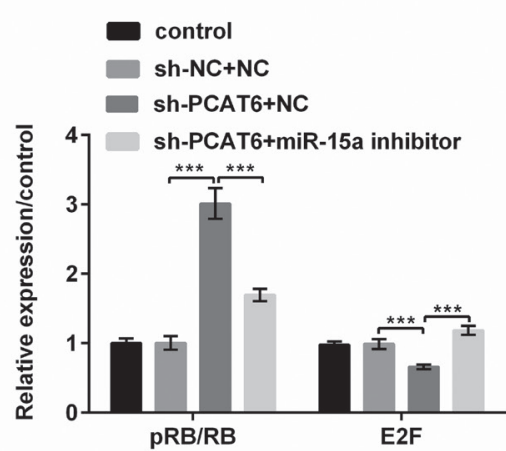

\section{E}

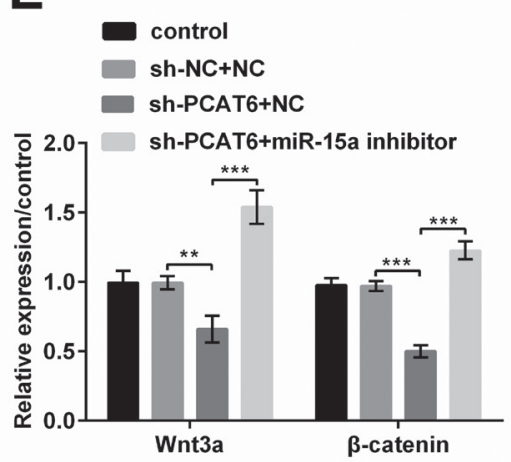

\section{G}

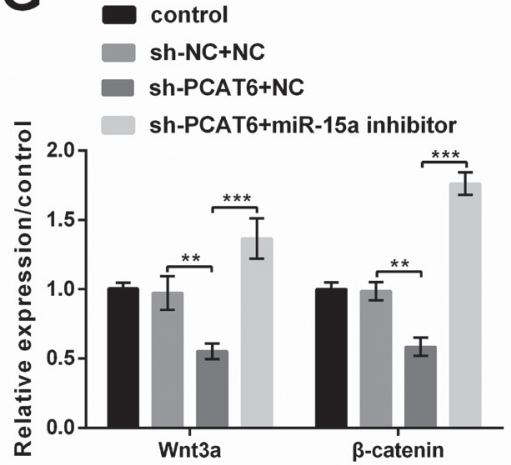

B

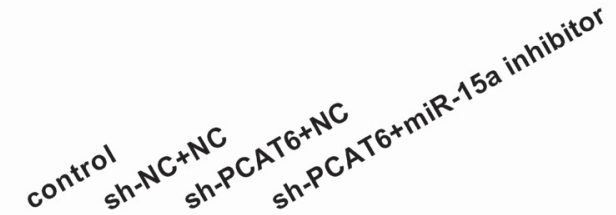

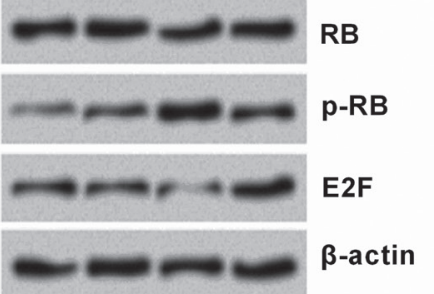

D
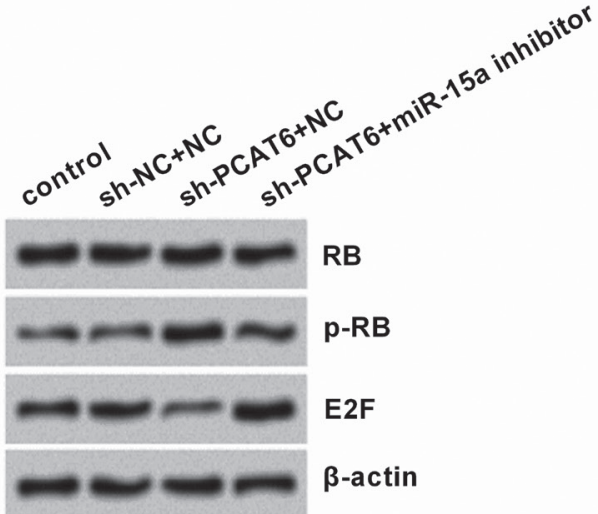

$\mathbf{F}$

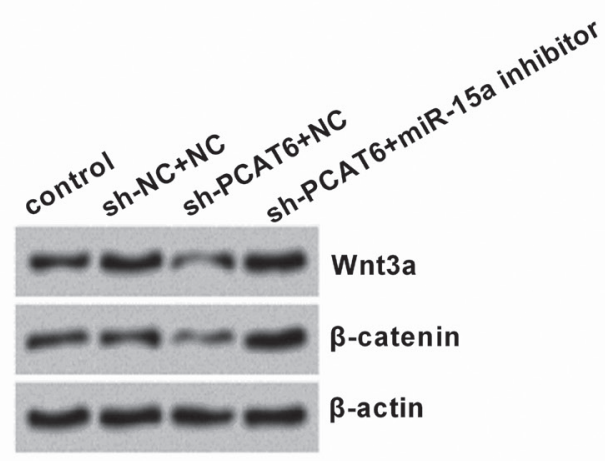

H

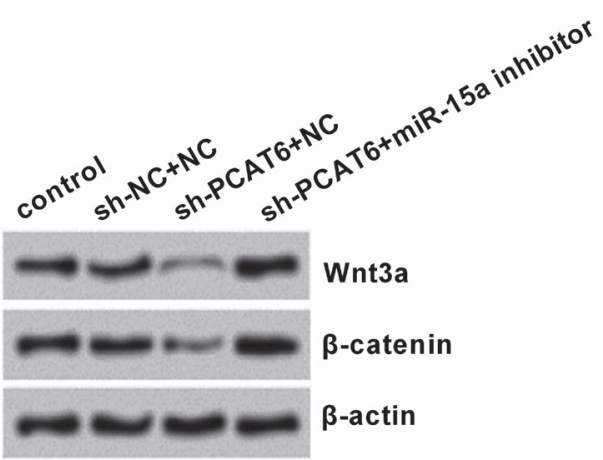

Figure 7. Silencing of PCAT6 restrained the RB/E2F and Wnt/ $\beta$ catenin signaling pathways via targeting miR-15a. Silencing of PCAT6 restrained the RB/E2F signaling pathway via targeting miR-15a in MKN45 cells (A and B) and in SGC-7901 cells $(\mathbf{C}$ and $\mathbf{D})$. Silencing of PCAT6 restrained the $\mathrm{Wnt} / \beta$-catenin signaling pathway via targeting miR-15a in MKN45 cells (E and F) and in SGC-7901 cells (G and H). ${ }^{* *} p<0.01 ;{ }^{* * *} p<0.001$. 


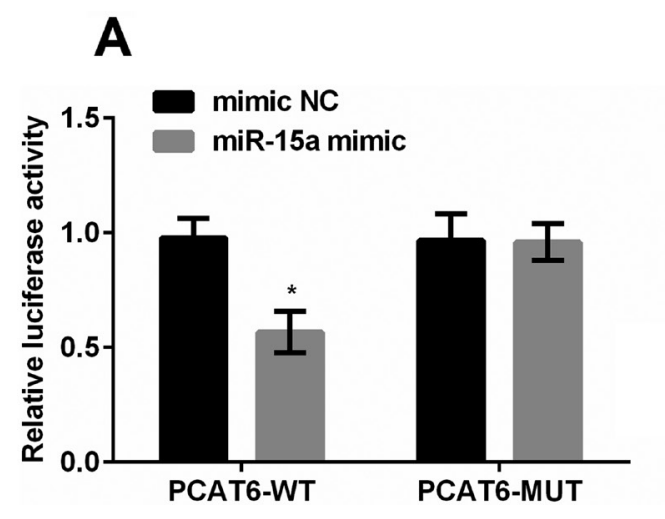

B
miR-15a 3'-...CGUCGUGUU...AUACCGGACGUUC...-5'
PCAT6 5'-.. CUUGGACAA...CCUGGCCUCC
5'-...CUUGGACAA...CCUGGCCUCCGGU...-3'

Figure 8. miR-15a was a target of PCAT6. A. The relationship between miR-15a and PCAT6 were detected by luciferase assay. B. The targeting sequence. ${ }^{\star} p<0.05$.

involved in GC through $\mathrm{RB} / \mathrm{E} 2 \mathrm{~F}$ and $\mathrm{Wnt} / \beta$-catenin signaling pathways (Ofir et al. 2011; Bai et al. 2019). Fortunately, we also discovered that the ratio of $\mathrm{pRB} / \mathrm{RB}$ was enhanced and the level of E2F, Wnt and $\beta$-catenin were declined when transfected sh-PCAT6, and these level were reversed when transfected with miR-15 inhibitor in this system. In short, silencing of PCAT6 restrained RB/E2F and Wnt/ $\beta$-catenin signaling pathways by the up-regulation of miR-15a.

In summary, silencing of lncRNA PCAT6 restrained proliferation and EMT of GC cells by targeting miR-15a via $\mathrm{RB} / \mathrm{E} 2 \mathrm{~F}$ and $\mathrm{Wnt} / \beta$-catenin signaling pathways. This article might afford a new thought for the clinical treatment of GC.

Acknowledgements. The research was encouraged by the Medical Ethics Committee of No.971 Hospital of People's Liberation Army Navy.

Conflict of interest. The authors declare that they have no conflict of interest.

Data availability statement. The datasets used and/or analyzed during the current study are available from the corresponding author on reasonable request.

\section{References}

Bai C, Zhang H, Zhang X, Yang W, Li X, Gao Y (2019): MiR-15/16 mediate crosstalk between the MAPK and Wnt/beta-catenin pathways during hepatocyte differentiation from amniotic epithelial cells. Biochim. Biophys. Acta 1862, 567-581 https://doi.org/10.1016/j.bbagrm.2019.02.003

Bi WR, Yang CQ, Shi Q (2012): Transforming growth factor- $\beta 1$ induced epithelial-mesenchymal transition in hepatic fibrosis. Hepat-gastroenterology 59, 1960-1963 https://doi.org/10.5754/hge11750

Birkenkamp-Demtroder K, Maghnouj A, Mansilla F, Thorsen K, Andersen CL, Oster B, Hahn S, Orntoft TF (2011): Repression of KIAA1199 attenuates Wnt-signalling and decreases the proliferation of colon cancer cells. Br. J. Cancer 105, 552-561 https://doi.org/10.1038/bjc.2011.268

Chen S, Chen Y, Qian Q, Wang X, Chang Y, Ju S, Xu Y, Zhang C, Qin N, Ding H, et al. (2019): Gene amplification derived a cancer-testis long noncoding RNA PCAT6 regulates cell proliferation and migration in hepatocellular carcinoma. Cancer Med. 8, 3017-3025

https://doi.org/10.1002/cam4.2141

Cimmino A, Calin GA, Fabbri M, Iorio MV, Ferracin M, Shimizu M, Wojcik SE, Aqeilan RI, Zupo S, Dono M, et al. (2005): miR15 and miR-16 induce apoptosis by targeting BCL2. Proc. Natl. Acad. Sci. USA 102, 13944-13949 https://doi.org/10.1073/pnas.0506654102

Cittelly DM, Das PM, Salvo VA, Fonseca JP, Burow ME, Jones FE (2010): Oncogenic HER2\{Delta\} 16 suppresses miR-15a/16 and deregulates BCL-2 to promote endocrine resistance of breast tumors. Carcinogenesis 31, 2049-2057 https://doi.org/10.1093/carcin/bgq192

Cui LH, Xu HR, Yang W, Yu LJ (2018): IncRNA PCAT6 promotes non-small cell lung cancer cell proliferation, migration and invasion through regulating miR-330-5p. OncoTargets Ther. 11, 7715-7724 https://doi.org/10.2147/OTT.S178597

Dong J, Qu Y, Li J, Cui L, Wang Y, Lin J, Wang H (2018): Cortisol inhibits NF-kB and MAPK pathways in LPS activated bovine endometrial epithelial cells. Int. Immunopharmacol. 56, 71-77 https://doi.org/10.1016/j.intimp.2018.01.021

Fabregat I, Fernando J, Mainez J, Sancho P (2014): TGF-beta signaling in cancer treatment. Cur. Pharma. Des. 20, 2934-2947 https://doi.org/10.2174/13816128113199990591

Hamashima C (2014): Current issues and future perspectives of gastric cancer screening. World Journal of Gastroenterology 20, $13767-13774$ https://doi.org/10.3748/wjg.v20.i38.13767

Hrovatin K, Kunej T (2018): Classification of miRNA-related sequence variations. Epigenomics 10, 463-481 https://doi.org/10.2217/epi-2017-0126 
Huang W, Su G, Huang X, Zou A, Wu J, Yang Y, Zhu Y, Liang S, Li D, Ma F, Guo L (2019): Long noncoding RNA PCAT6 inhibits colon cancer cell apoptosis by regulating anti-apoptotic protein ARC expression via EZH2. Cell Cycle 18, 69-83 https://doi.org/10.1080/15384101.2018.1558872

Jarroux J, Morillon A, Pinskaya M (2017): History, discovery, and classification of lncRNAs. Adv. Exp. Med. Biol. 1008, 1-46 https://doi.org/10.1007/978-981-10-5203-3_1

Kajdaniuk D, Marek B, Borgiel-Marek H, Kos-Kudla B (2013): Transforming growth factor $\beta 1$ (TGF $\beta 1$ ) in physiology and pathology. Endokrynol. Pol. 64, 384-396 https://doi.org/10.5603/EP.2013.0022

Kang W, Tong JH, Lung RW, Dong Y, Zhao J, Liang Q, Zhang L, Pan Y, Yang W, Pang JC, et al. (2015): Targeting of YAP1 by microRNA-15a and microRNA-16-1 exerts tumor suppressor function in gastric adenocarcinoma. Mol. Cancer 14, 52 https://doi.org/10.1186/s12943-015-0323-3

Karimi P, Islami F, Anandasabapathy S, Freedman ND, Kamangar F (2014): Gastric cancer: descriptive epidemiology, risk factors, screening, and prevention. Cancer Epidemiol. Biomarkers Prev. 23, 700-713 https://doi.org/10.1158/1055-9965.EPI-13-1057

Kim HM, Haraguchi N, Ishii H, Ohkuma M, Okano M, Mimori K, Eguchi H, Yamamoto H, Nagano H, Sekimoto M, et al. (2012): Increased CD13 expression reduces reactive oxygen species, promoting survival of liver cancer stem cells via an epithelial-mesenchymal transition-like phenomenon. Ann. Surg. Oncol. 19, S539-548

https://doi.org/10.1245/s10434-011-2040-5

Ling H (2016): Non-coding RNAs: Therapeutic strategies and delivery systems. Adv. Exp. Med. Biol. 937, 229-237 https://doi.org/10.1007/978-3-319-42059-2_12

Lordick F, Shitara K, Janjigian YY (2017): New agents on the horizon in gastric cancer. Ann. Oncol. 28, 1767-1775 https://doi.org/10.1093/annonc/mdx051

Ma L, Zhou Y, Luo X, Gao H, Deng X, Jiang Y (2017): Long noncoding RNA XIST promotes cell growth and invasion through regulating miR-497/MACC1 axis in gastric cancer. Oncotarget 8, 4125-4135 https://doi.org/10.18632/oncotarget.13670

Misawa A, Takayama KI, Inoue S (2017): Long non-coding RNAs and prostate cancer. Cancer Sci. 108, 2107-2114 https://doi.org/10.1111/cas.13352

Musumeci M, Coppola V, Addario A, Patrizii M, Maugeri-Sacca M, Memeo L, Colarossi C, Francescangeli F, Biffoni M, Collura D, et al. (2011): Control of tumor and microenvironment cross-talk by miR-15a and miR-16 in prostate cancer. Oncogene 30, 4231-4242 https://doi.org/10.1038/onc.2011.140

Mutlu M, Raza U, Saatci O, Eyupoglu E, Yurdusev E, Sahin O (2016): miR-200c: a versatile watchdog in cancer progression, EMT, and drug resistance. J. Mol. Med. 94, 629-644 https://doi.org/10.1007/s00109-016-1420-5

Nasrollahzadeh-Khakiani M, Emadi-Baygi M, Schulz WA, Nikpour P (2017): Long noncoding RNAs in gastric cancer carcinogenesis and metastasis. Brief. Funct. Genomics 16, 129-145 https://doi.org/10.1093/bfgp/elw011
O'Leary K, Shia A, Schmid P (2018): Epigenetic regulation of EMT in non-small cell lung cancer. Curr. Cancer Drug Targets 18, 89-96 https://doi.org/10.2174/1568009617666170203162556

Ofir M, Hacohen D, Ginsberg D (2011): MiR-15 and miR-16 are direct transcriptional targets of E2F1 that limit E2F-induced proliferation by targeting cyclin E. Mol. Cancer Res. 9, 440-447 https://doi.org/10.1158/1541-7786.MCR-10-0344

Pillai S, Trevino J, Rawal B, Singh S, Kovacs M, Li X, Schell M, Haura E, Bepler G, Chellappan S (2015): beta-arrestin-1 mediates nicotine-induced metastasis through E2F1 target genes that modulate epithelial-mesenchymal transition. Cancer Res. 75, $1009-1020$ https://doi.org/10.1158/0008-5472.CAN-14-0681

Schwarzenbach H (2016): Biological and clinical relevance of H19 in colorectal cancer patients. EBioMedicine 13, 9-10 https://doi.org/10.1016/j.ebiom.2016.11.001

Shi X, Liu Z, Liu Z, Feng X, Hua F, Hu X, Wang B, Lu K, Nie F (2018): Long noncoding RNA PCAT6 functions as an oncogene by binding to EZH2 and suppressing LATS2 in non-small-cell lung cancer. EBioMedicine 37, 177-187 https://doi.org/10.1016/j.ebiom.2018.10.004

Shin VY, Chu KM (2014): MiRNA as potential biomarkers and therapeutic targets for gastric cancer. World J. Gastroenterol. 20, 10432-10439 https://doi.org/10.3748/wjg.v20.i30.10432

Singh AS, Heery R, Gray SG (2018): In silico and in vitro analyses of lncRNAs as potential regulators in the transition from the epithelioid to sarcomatoid histotype of malignant pleural mesothelioma (MPM). Int. J. Mol. Sci. 19, E1297 https://doi.org/10.3390/ijms19051297

Song J, Yin J, Bai Z, Zhang J, Meng H, Cai J, Deng W, Ma X, Zhang $Z$ (2017a): The profile of serum microRNAs predicts prognosis for resected gastric cancer patients receiving platinum-based chemotherapy. Dig. Dis. Sci. 62, 1223-1234 https://doi.org/10.1007/s10620-017-4513-2

Song Z, Wu Y, Yang J, Yang D, Fang X (2017b): Progress in the treatment of advanced gastric cancer. Tumour Biol. 39, 1010428317714626 https://doi.org/10.1177/1010428317714626

Takeshita F, Patrawala L, Osaki M, Takahashi RU, Yamamoto Y, Kosaka N, Kawamata M, Kelnar K, Bader AG, Brown D, Ochiya $\mathrm{T}$ (2010): Systemic delivery of synthetic microRNA-16 inhibits the growth of metastatic prostate tumors via downregulation of multiple cell-cycle genes. Mol. Ther. 18, 181-187 https://doi.org/10.1038/mt.2009.207

Tsukamoto T, Nakagawa M, Kiriyama Y, Toyoda T, Cao X (2017): Prevention of gastric cancer: Eradication of Helicobacter Pylori and beyond. Int. J. Mol. Sci. 18, 1699 https://doi.org/10.3390/ijms18081699

Wan L, Zhang L, Fan K, Cheng ZX, Sun QC, Wang JJ (2016): Knockdown of long noncoding RNA PCAT6 inhibits proliferation and invasion in lung cancer cells. Oncol. Res. 24, 161-170 https://doi.org/10.3727/096504016X14618564639178

Wan L, Zhang L, Fan K, Wang JJ (2017): Diagnostic significance of circulating long noncoding RNA PCAT6 in patients with non-small cell lung cancer. Onco Targets Ther. 10, 5695-5702 https://doi.org/10.2147/OTT.S149314 
Wang Z, Wang Q, Wang C, Xu X, Yu H (2017): Tetramethylpyrazine attenuates periorbital allodynia and neuroinflammation in a model of traumatic brain injury. J. Inflamm. 14, 13 https://doi.org/10.1186/s12950-017-0161-8

Wu C, Zheng X, Li X, Fesler A, Hu W, Chen L, Xu B, Wang Q, Tong A, Burke S, Ju J, Jiang J (2016): Reduction of gastric cancer proliferation and invasion by miR-15a mediated suppression of Bmi-1 translation. Oncotarget 7, 14522-14536 https://doi.org/10.18632/oncotarget.7392

Wu H, Zou Q, He H, Liang Y, Lei M, Zhou Q, Fan D, Shen L (2019) Long non-coding RNA PCAT6 targets miR-204 to modulate the chemoresistance of colorectal cancer cells to 5 -fluorouracilbased treatment through HMGA2 signaling. Cancer Med. 8, 2484-2495

https://doi.org/10.1002/cam4.1809
Xia T, Liao Q, Jiang X, Shao Y, Xiao B, Xi Y, Guo J (2014): Long noncoding RNA associated-competing endogenous RNAs in gastric cancer. Sci. Rep. 4, 6088 https://doi.org/10.1038/srep06088

Xu Y, Sun JY, Jin YF, Yu H (2018): PCAT6 participates in the development of gastric cancer through endogenously competition with microRNA-30. Eur. Rev. Med. Pharmacol. Sci. 22, 5206-5213

Zhou X, Ye F, Yin C, Zhuang Y, Yue G, Zhang G (2015): The interaction between MiR-141 and lncRNA-H19 in regulating cell proliferation and migration in gastric cancer. Cell. Physiol. Biochem. 36, 1440-1452 https://doi.org/10.1159/000430309

Received: May 23, 2019

Final version accepted: October 2, 2019 